\title{
A flexible adaptive optics concept for general purpose high angular resolution science on the DAG $4 \mathrm{~m}$ telescope
}

Laurent Jolissaint, Audrey Bouxin, Ulaş S. Gökay, Onur Keskin, François Rigaut, et al.

Laurent Jolissaint, Audrey Bouxin, Ulaş S. Gökay, Onur Keskin, François Rigaut, Cahit Yesilyaprak, "A flexible adaptive optics concept for general purpose high angular resolution science on the DAG 4m telescope," Proc. SPIE 10703, Adaptive Optics Systems VI, 1070366 (11 July 2018); doi: 10.1117/12.2313655

EDIE Event: SPIE Astronomical Telescopes + Instrumentation, 2018, Austin, Texas, United States 


\title{
A flexible adaptive optics concept for general purpose high angular resolution science on the DAG $4 \mathrm{~m}$ telescope
}

\author{
Laurent Jolissaint ${ }^{\mathrm{a}}$, Audrey Bouxin ${ }^{\mathrm{a}}$, Ulaş S. Gökay ${ }^{\mathrm{b}}$, Onur Keskin ${ }^{\mathrm{b}}$, François Rigaut ${ }^{\mathrm{c}}$, and \\ Cahit Yesilyaprak ${ }^{\mathrm{d}}$ \\ ${ }^{a}$ University of Applied Science Western Switzerland, School of Business and Engineering, Rte. \\ de Cheseaux 1, CH-1401, Yverdon-les-Bains, Switzerland \\ ${ }^{\mathrm{b}}$ FMV Işı University, Center for Optomechatronics Research \& Application - OPAM, \\ Istanbul, Turkey \\ ${ }^{c}$ Research School of Astronomy \& Astrophysics, Australian National University, Canberra, \\ Australia \\ ${ }^{\mathrm{d} A t a t u ̈ r k}$ University Application and Research Center for Astrophysics (ATASAM), Erzurum, \\ Turkey
}

\begin{abstract}
Thanks to the availability of high actuator density deformable mirrors (ALPAO $468 \mathrm{DM}$ ), the high versatility of the pyramid wavefront sensor and above all, the venue of essentially no noise EMCCD detectors, it becomes possible to fully adapt the degree of correction of an adaptive optics system for a given guide star brightness and atmospheric condition. Indeed, when the conditions are very good, the high actuator density of the DM allows to reach a high Strehl by using all the modes, and when the conditions are less favorable, the spatial sampling, i. the number of modes, and the sensitivity of the detector allows to maximize the Strehl beyond what would be possible with a classical, frozen SH-WFS based system. Beside, oversampling the detector allows to relax the specification on the pupil images given by the pyramid on the detector, which in turn relaxes the pyramid prism manufacturing specifications. We are now designing an AO system for the DAG $4 \mathrm{~m}$ telescope that will allow, on the same system, ExAO as well as low order improved seeing observations. This article reports on the AO performance analysis, the final optical design and the design of the double prism achromatic pyramid.
\end{abstract}

Keywords: Adaptive optics, pyramid wavefront sensor, flexible control

\section{INTRODUCTION}

We present in this proceeding the current status of the development of the adaptive optics system (AO) of DAG, the future $4 \mathrm{~m}$ Turkish telescope. ${ }^{1}$ DAG-AO is a natural guide star (NGS) and pyramid wavefront sensor (PWFS) based system. ${ }^{2}$ The deformable mirror has a high density of actuators, more than what a classical system would require, in order to be able to benefit from the flexibility offered by the P-WFS sensitivity and pupil spatial sampling adjustments.

In this paper, we present the flexible $\mathrm{AO}$ concept that we aim for DAG-AO, the performance estimations using an end-to-end $\mathrm{AO}$ simulation software, the current design of the optical system, and the pyramid prism design.

Further author information: (Send correspondence to Laurent Jolissaint) laurent.jolissaint@heig-vd.ch, Telephone: +41245576421 


\section{THE FLEXIBLE AO CONCEPT}

The reasons why we have developed the flexible concept is presented in Jolissaint et al.. ${ }^{3}$ In short, the objective is to offer to the community of the future users of DAG AO the most possible versatile system when using only NGS, allowing extreme level of correction when the seeing and NGS conditions permit (ExAO), but also the best possible level of correction otherwise, in an SCAO mode.

For a moderate 1" seeing, about 80 actuators are required to get a decent correction in the NIR (Strehl about $70 \%$ with a bright NGS, basically one actuator per $r_{0}$ length at the science wavelength). Instead, we will use a DM with 468 actuators (from ALPAO, France). If the conditions are favorable, i.e if the NGS is bright $(V \leq 10)$ and the seeing moderate to good (below 1") then we will use most of the actuators and get an ExAO type of correction (allowing for instance stellar coronagraphy at high rejection ratio). If the conditions are less favorable, then on the contrary we will lower the number of corrected modes, by playing with the individual modal gains and the integration time.

If we use a Shack-Hartmann system, the optimization stops here. But we will use a PWFS, first because it is known to be more efficient in photons starved regime, but mostly because the spatial sampling and the sensitivity can be adjusted as well, to always get the best signal/noise ratio for a given observation. In other words, the PWFS can always be adjusted to the number of modes we want to correct, optimizing the performance beyond what is possible with a SH-based system.

Five parameters will be adjusted in the flexible mode concept :

the wavefront sensor integration time, to balance the servo-lag and the WFS noise errors; this is classical in $\mathrm{AO}$;

the loop gain of each system's mode, for the same reason as above; this is also a classical procedure;

the pupil spatial sampling of the PWFS detector; when the NGS magnitude increases, the spatial sampling interval in the pupil plane should be decreased (binning the pixels), in order to keep a constant signal to noise ratio (SNR) on the wavefront measurement; while doing this, we also adapt the number of corrected modes, by changing the modal gains; so there will be a direct connexion between the modal gains and the spatial sampling of the pupil;

the modulation angle of the NGS beam on the apex of the pyramid; the lower the spatial frequency of the modes we want to correct, the larger the optimal modulation angle, so in dim NGS conditions, when we will correct only a few modes, we should use a larger modulation angle than in bright NGS conditions ;

the signal/noise regularization parameter of the MMSE algorithm ; we shall use the MMSE algorithm in the loop control of the loop, as it is one of the most efficient reconstruction method known as today; we still need to understand how we shall adapt this parameter as a function of the NGS and seeing conditions.

From the above, it is clear that all the 5 parameters are connected. An algorithm will have to be developed to find, for the given conditions, the best values of the parameters. Note that the seeing and NGS brightness can be extracted from the loop data, following the DM-seeing technique described in Jolissaint et al. ${ }^{4}$ and counting the number of counts in the WFS detector pixels. The scheme we are therefore proposing, shown in Fig. 1, is to implement a telemetry acquisition, from which the seeing and the NGS brightness can be extracted, and the best parameters found by the optimization algorithm (where machine learning might be of interest).

\section{Pupil images oversampling}

We will oversample the pupil images by a factor 1.5, in order to relax the constraints on the pupil images position, allowing in turn a loosening of the specifications of the pyramid faces angles, as proposed in Lardière et al.. ${ }^{5}$ This is made possible by the fact that we will use a virtually zero noise EM-CCD detector (NUVU 128 pixel EMCCD ${ }^{*}$ ). With such a camera, the pupil images can be resampled numerically after reading the image, allowing an arbitrary change of the spatial sampling, with no loss of information or extra noise.

\footnotetext{
${ }^{*}$ http ://www.nuvucameras.com
} 


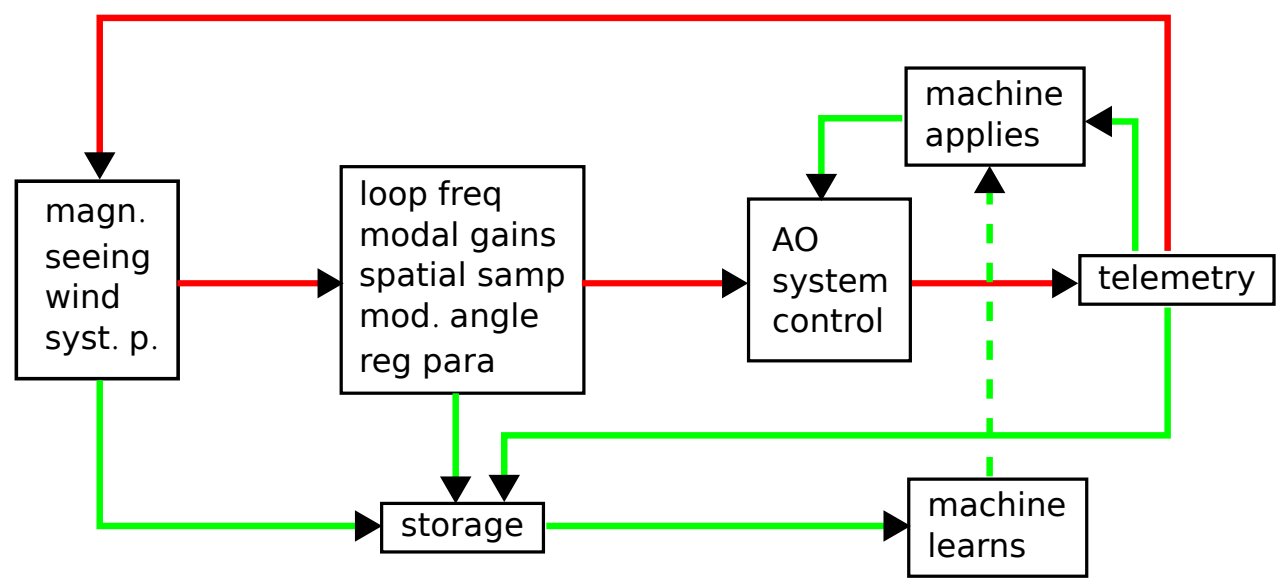

FIGURE 1. Overall scheme for the constant optimization of the AO loop parameters as a function of the NGS and atmospheric conditions.

\section{Machine learning and system optimization}

It is clear that the optimization algorithm will take some time to find the best solution amongst all the 5 parameters. It might therefore be interesting to explore what a machine learning algorithm could do to improve the refreshing rate of the parameters. This path is shown in green in Fig. 1. Potentially, such system could improve the correction quality. This remain to be evaluated.

\section{PERFORMANCE ANALYSIS}

Before developing any optimization algorithm, it is important to verify that an optimization is indeed worth doing, i.e if optimizing the system can bring any gain in performance with respect to a static case. To study this, we have simulated our system with YAO, an end-to-end Monte-Carlo AO system simulator (Rigaut \& van $\mathrm{Dam}^{6}$ ). Our objective was to evaluate the Strehl in the middle of the NIR bands, at $1.65 \mu \mathrm{m}$, for a range of NGS V band magnitudes, from 4 to 18, and optimize all the five parameters. These results, while preliminary, are showing what should be the envelope performance of the Strehl as a function of the NGS magnitude. Note that there is no modal gain optimization in the mode, only an overall gain optimization, and that the loop delay is conservatively set to 2 frames.

\subsection{YAO model parameters range}

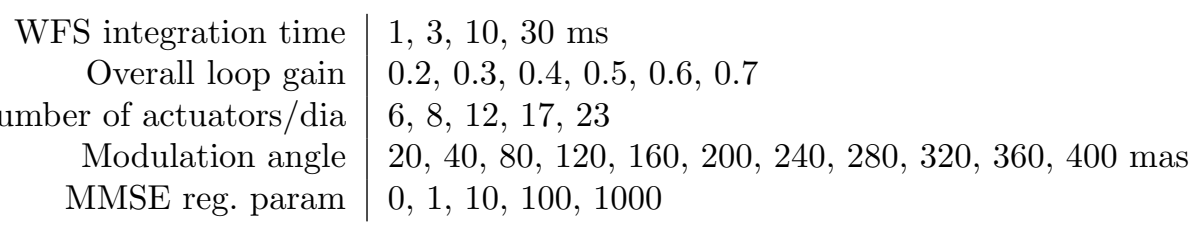

The other constant parameters of the simulation are

\begin{tabular}{r|l} 
telescope diameters & $3940 \& 975 \mathrm{~mm}$ \\
$r_{0}$ at $500 \mathrm{~nm}$ & $0.12 \mathrm{~m}$ \\
$C_{N}^{2}$ distribution & 6 layers, $40 \%$ at ground level \\
$r_{0}$ sampling & 3 pixels $/ r_{0}$ at $500 \mathrm{~nm}$ \\
WFS central $\lambda$ & $650 \mathrm{~nm}$ \\
average wind velocity & $10 \mathrm{~m} / \mathrm{s}$ \\
time lag (read/CPU) & equal to the WFS int time
\end{tabular}


There are of course many parameters whose impact we still have to study, for instance the effect of the misregistration between the DM and the WFS, the sky background (Moon) effect, and others. Beside, the precise atmospheric parameters at the site (Karakaya summit) are still uncertain, even if preliminary DIMM measurements have shown that the median seeing is 0.87 ".

\subsection{Overall performance}

We understand when looking at the results that the sensitivity of the Strehl performance to a given parameter strongly depends on the value of the other parameters. For instance, when we use all the actuators of the DM (468 except a few off the pupil), we can see from Fig. 2, left, that changing the WFS integration time does not help in optimizing the Strehl for a given magnitude : it is only by changing the overall loop gain that we can maximize the Strehl.

Also, we can see that indeed, it is possible to adjust the parameters to improve the Strehl at each magnitude, but sometime the gain is only at high magnitude and low Strehl, and trying to optimize the system at very low Strehl has no interest. In other words, optimization is one nice thing, but in order to be interesting it has to occur in conditions where the Strehl has already reached some value. Increasing the Strehl by a factor 10 when it is equal to 0.001 is useless!

Fig. 2, right, shows the best Strehl envelope when optimizing the loop gain, the WFS integration time, and the number of corrected modes. Decreasing the number of modes allows to gain 1.5 magnitudes while keeping a decent correction (say at least $40 \%$ Strehl). This is encouraging. The same figure shows that with less that 11 actuators across the pupil, the high magnitude performance does not improve. This is surprising, but the modulation angle was not optimized for these simulations and this might be an explanation.

Overall, this E2E simulation validate the best performance predictions we did in our previous article,$^{3}$ in an ExAO mode (based on a SH WFS, but at high Strehl the performance difference with the PWFS should be marginal). Therefore, we shall be able, if the non common path aberrations are well managed and the site is as good as expected, to produce Strehl above $90 \%$ in H-band, for NGS magnitudes up to V=10. And when the conditions will be less favorable, the system can work as a classical moderate order AO by reducing the number of corrected modes, and optimizing.

\subsection{About the modulation angle effect}

It is known ${ }^{7}$ that when the low order WFE get stronger, the sensitivity of the PWFS to the low orders can be increased by adjusting the modulation angle. This is shown in Fig. 3, left, where we have increased the seeing angle from 0.3" to 1.5" and computed the Strehl for a modulation angle in the range 20 to 400 mas. The green curve shows how the optimal modulation angle increases with the seeing. The well known fact that the better the correction, the lower the modulation can be is well illustrated. It clearly demonstrates also that the system will definitely have to allow an adjustment of the modulation angle, as not doing so would led to clearly suboptimal performances in some cases.

Fig. 3, right, shows the modulation angle optimization for a set of magnitudes and actuators density, for a seeing of $0.84 "$ ". Again there is an obvious interest to optimize the angle, particularly when the noise is high. It does not seem that the optimal angle changes with the noise level, but this simulation is relatively coarse, and our objective was mostly to explore the relationship between noise and modulation.

\subsection{To conclude}

The flexible concept presents an obvious interest. ExAO as well as classical SCAO shall be possible, with the same system. The whole optimization loop, as shown in Fig. 1, with possible machine learning, still has to be developed. It is important to note that such a scheme would not have been possible without the availability of high actuators density DM, pyramid WFS, and very low noise CCD camera. 

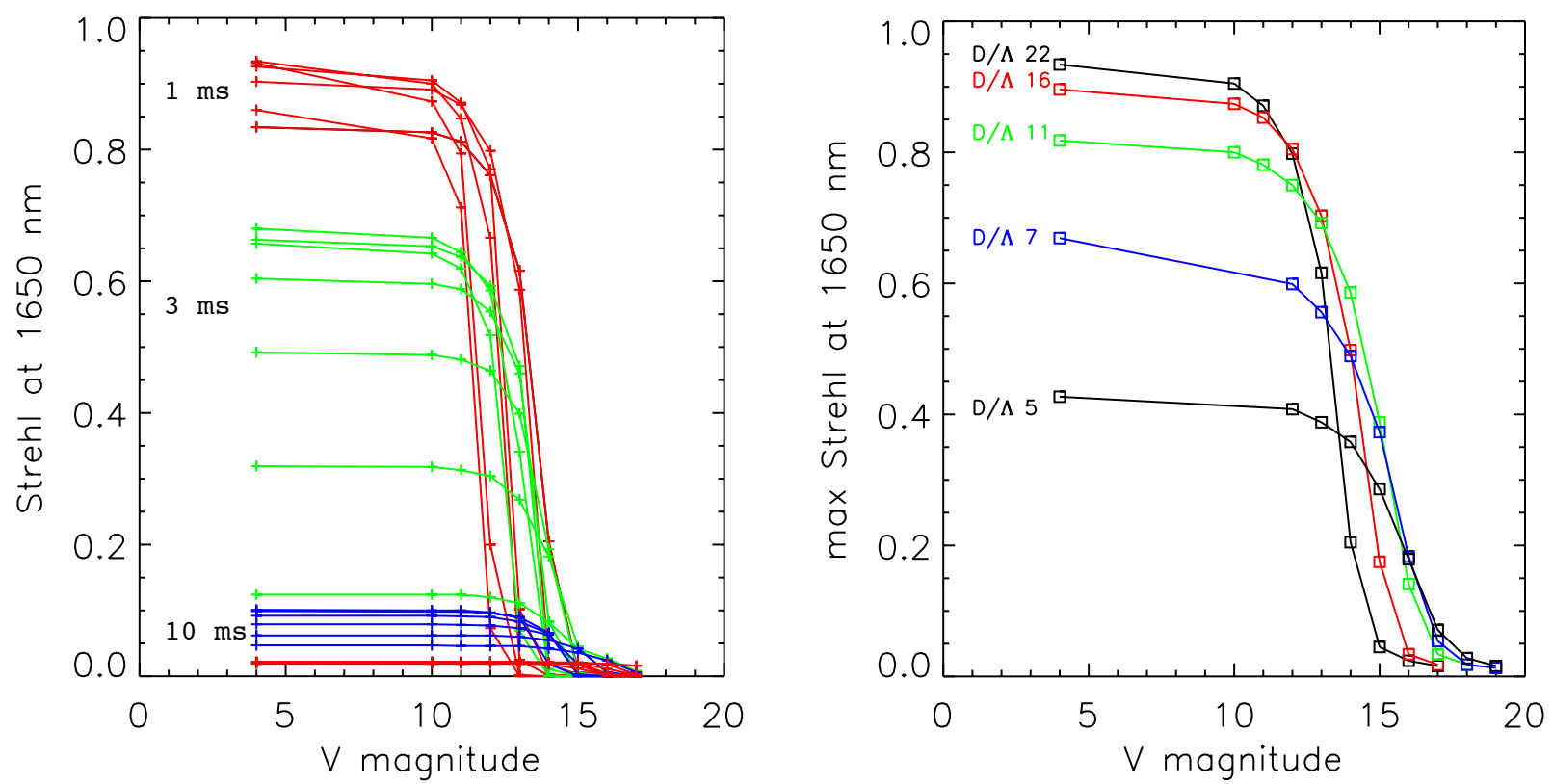

FIGURE 2. Left : Full DM density (23 actuators across the DM). Strehl as a function of the NGS magnitude for an overall loop gain from 0.2 to 0.7 and a WFS integration time of $1 \mathrm{~ms}, 3 \mathrm{~ms}, 10 \mathrm{~ms}$ and $30 \mathrm{~ms}$. Right : Best Strehl envelope for an increasing density of actuators. The loop gain was varied from 0.2 to 0.7 and the WFS integration time from 1 to $30 \mathrm{~ms}$.
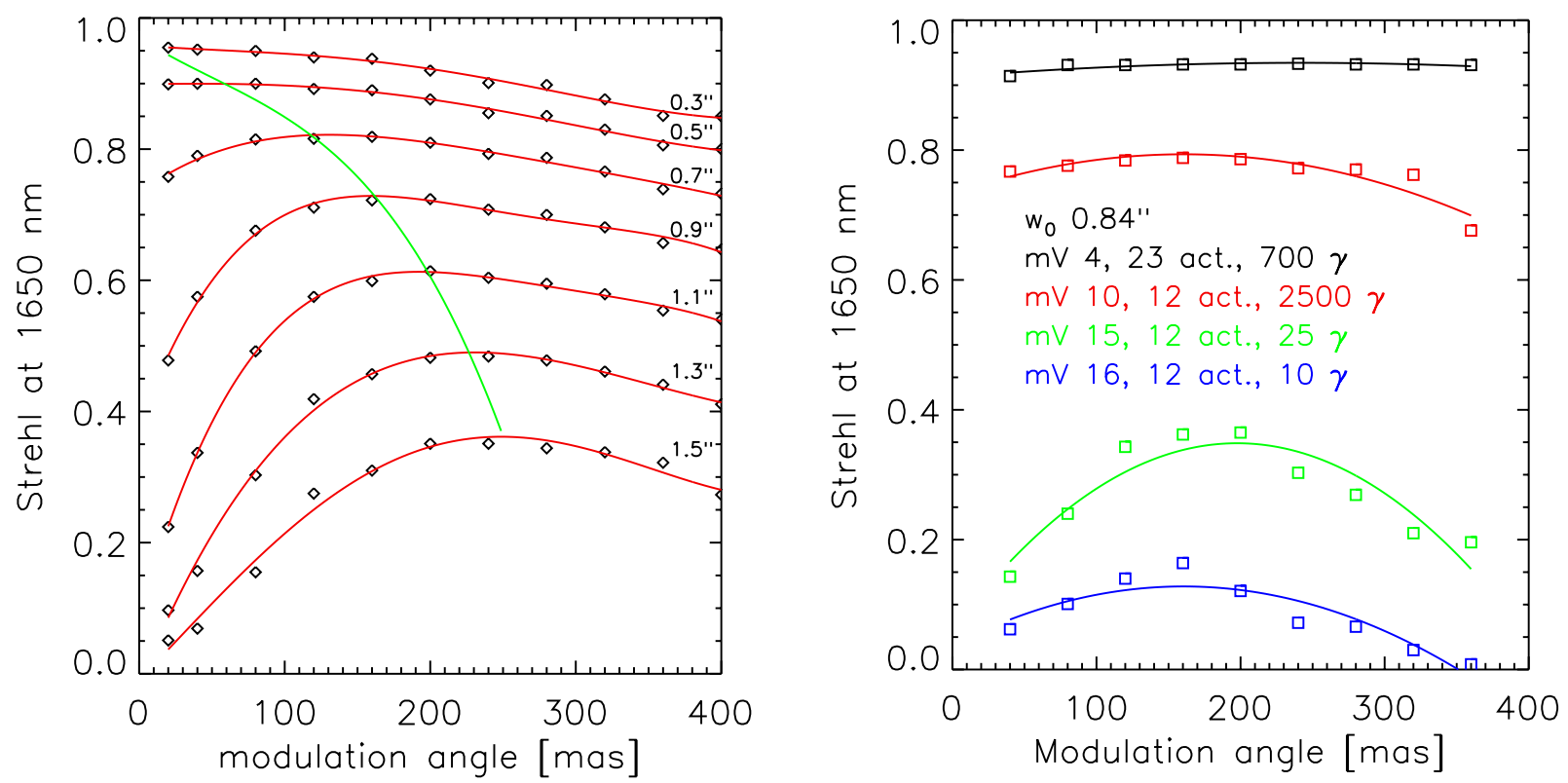

FiguRE 3. Left : Modulation angle optimization for an increasing seeing, from 0.3" to 1.5" (best/worst expected at the site). Red curves are 4th order polynomials fitting. Right : Modulation angle optimization for a decreasing number of photons, from 10 to $2500 \mathrm{\gamma} / \mathrm{pixel} /$ frame. 


\section{DESIGN OF THE ADAPTIVE OPTICS SYSTEM}

This section presents DAG AO optical train design and optimization. The principle is to minimize the number of optical surfaces and to design a compact system. The optical surfaces are designed for a 1' scientific FoV (Field of view) and 8" WFS (Wavefront Sensor) FoV. The design constraints are the following :

- the pupil has to be imaged on the DM;

— the DM diameter is defined by the ALPAO DM-468 clear aperture Ø33 mm;

- the pupil has to be imaged on a TT (Tip-Tilt) mirror for beam modulation on the apex of the pyramid;

- the beam has to be focused on the pyramid apex;

- the angle of the beam onto the pyramid apex is calculated in order to get a diffraction limited PSF size of at least 2 times the pyramid residual roof due to manufacturing limitations;

- the pupil has to be imaged onto the NUVU EMCCD detector;

- the beam footprint diameter onto the detector has to be set accordingly to the oversampling criterion (see section 4.4).

The constraints parameters values are given below

$$
\begin{array}{r|l}
\text { telescope exit pupil diameter } & \emptyset_{\text {ExtP }}=727.4046 \mathrm{~mm} \\
\text { distance from exit pupil to focal plane } & \overline{\operatorname{ExP}-\mathrm{FP}}=10338.74 \mathrm{~mm} \\
\text { ALPAO DM-468 clear aperture diameter } & \emptyset_{\mathrm{DM}}=33.0 \mathrm{~mm} \\
\text { ALPAO DM-468 pitch } & \Lambda=1.5 \mathrm{~mm} \\
\text { pixel size of the NUVU EMCCD AO } & \text { PxSize }=24 \mu \mathrm{m} .
\end{array}
$$

There are 22 actuator's pitch across the DM diameter, and 23 actuators from edge to edge.

The design is split in four sections. All the design and optimizations have been made using the optical software ZEMAX.

\subsection{Imaging the exit pupil of the telescope onto the deformable mirror}

The first section is the system to image the telescope pupil onto the DM. The beam footprint has to fit the entire clear aperture of the DM. The maximum acceptable angle of the DM is calculated such as to have a registration error due to the elliptical beam footprint of maximum $1 / 10^{t h} \Lambda$. The imaging system consists of an OAP (Off-Axis Parabola, OAP0 in Fig. 4) that collimates the beam and images the pupil. Moreover, having a collimated beam at the entrance or output of the OAP limits the spherical aberrations. The OAP has two functions : (1) image the pupil, (2) fold the beam.

\subsection{Imaging the pupil on the tip-tilt modulation mirror and focus on the pyramid apex}

We need to image the pupil on the TT modulation mirror. Moreover, to compensate for the aberration introduced by OAP0 we can use an OAP1 with the same focal length. The F/D ratio would stay the same as the telescope's, a favorable situation for the science beam. In order to avoid to send back the beam on the focal plane and because we need to position the dichroic near the intermediate focal plane, we fold the beam after the DM with a flat mirror.

To minimize the dichroic membre, it is positionned near the intermediate focal plane. A folding mirror is added to send the light in the direction of the instruments. This allows avoiding a large incidence angle on the dichroic which would generate a large reflection loss due to polarization.

As the OAP0 and OAP1 are identical, the optical configuration creates an image of the pupil of the same diameter at the same distance from the focal plane as the exit pupil. But we want to have a beam of $10 \mathrm{~mm}$ in diameter at the pupil plane on the TT mirror and we also need to focus the beam on the pyramid roof (PWFS), with the following constraint :

$$
\text { PSF FWHM }=\lambda F / D>2 \text { pyramid roof }
$$




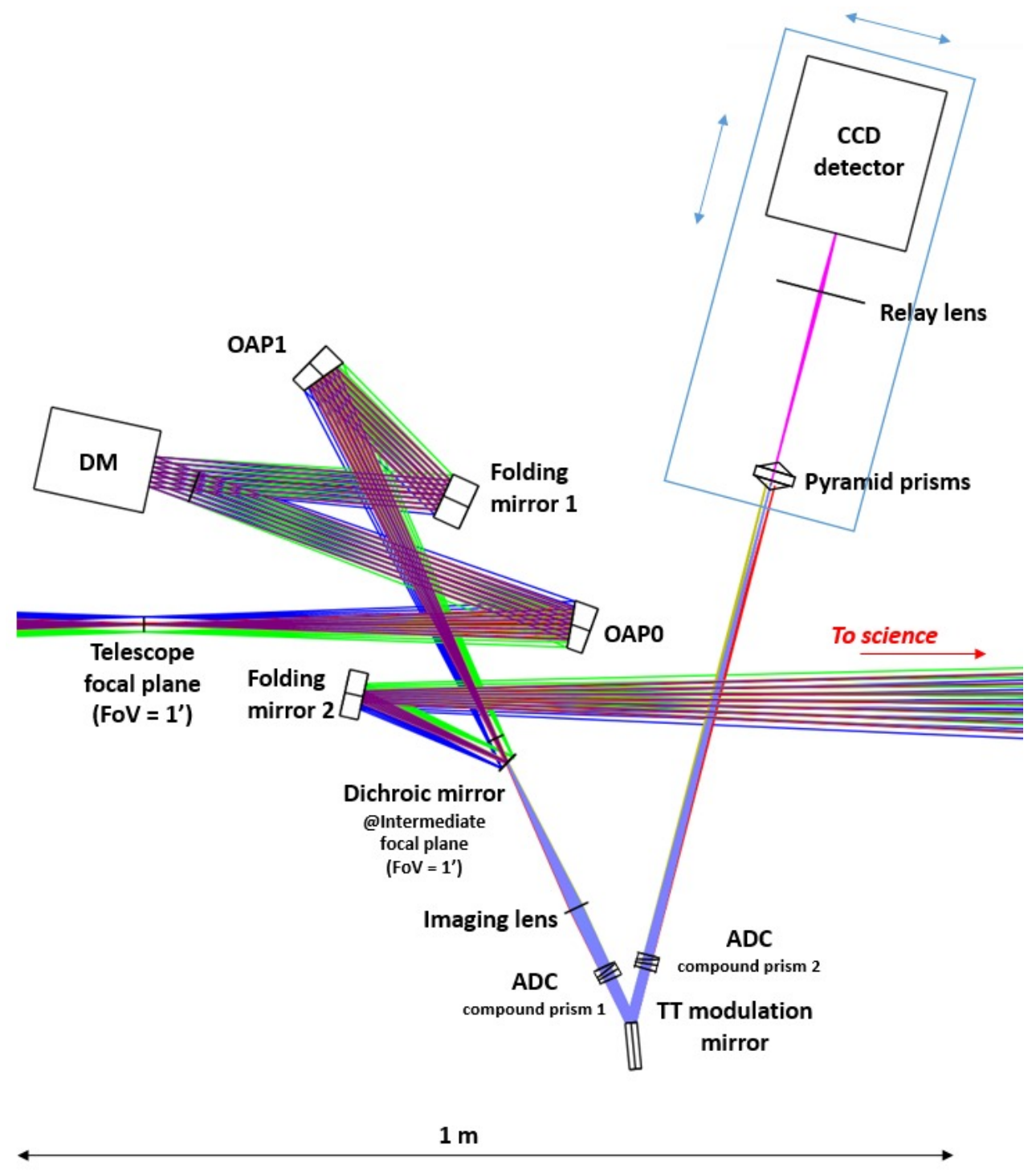

Figure 4. ZEMAX model of the AO system

Considering that a pyramid roof of about $20 \mu \mathrm{m}$ at $\lambda=0.6 \mu \mathrm{m}$ can be relatively easily achieved (Jean-Pierre Véran, priv. comm.), we find that the $\mathrm{F} / \mathrm{D}$ ratio must be $>66.7$. We round this value to $\mathrm{F} \#=f / \mathrm{D}=60$, but we need to investigate the minimum pyramid roof size that can actually be manufactured.

We want then a lens power that takes the beam with a F\# of 14 and brings it to 60 . The TT modulation mirror is placed in this converging beam at the pupil location. We choose an imaging doublet lens because the wavelength range in the WFS path is $500 \mathrm{~nm}$ to $900 \mathrm{~nm}$ and achromatism can be easily compensated. Its focal length is fixed to $f=140 \mathrm{~mm}$.

\subsection{Compensating for the atmospheric dispersion}

In order to correct for the chromatic dispersion of the atmosphere we need to introduce an ADC (Atmospheric Dispersion Compensator) in our system. It has to be as close as possible to a pupil plane, and to avoid having to reimage the pupil and add other optical surfaces we decided to insert the two parts of the ADC around the TT modulation mirror. 


\subsection{Imaging the pupil on the CCD detector}

The detector has a pixel size of $24 \mu \mathrm{m}$. We have to sample the beam with at least 1 pixel per actuator. In order to relax the alignment specifications we can oversample the beam with a factor of 1.5 (Jean-Pierre Véran, pers. comm.). ALPAO DM-468 has 22 actuators pitch across the clear aperture diameter. The beam diameter on the detector therefore has to be :

$$
\varnothing_{\mathrm{CCD}}=\#_{\text {actuator across } \varnothing} \times \text { PxSize } \times \text { Oversampling factor }=0.792 \mathrm{~mm}
$$

The pupil has to be imaged on the camera through a relay lens. We choose its focal length in order to have a lens that can easily be manufactured, or found in optical companies catalogue. Playing with this parameter we found that a focal of $60 \mathrm{~mm}$ would be a good choice.

The NGS selection in the FoV is made using an XYZ stage on which the PWFS is mounted. The TT modulation mirror is on a pupil plane so it can modulate the beam around the apex of the pyramid irrespectively of the star position. However the pyramid has to be placed at the star focus in the FoV.

\subsection{Optimization results}

The AO optical model on ZEMAX shows that if the alignment and optical surfaces are perfect we should have a total amount of wavefront aberration of less than $1 \mathrm{~nm}$ (largely dominated by spherical aberrations). The system wavefront aberration will therefore be limited by the optical elements manufacturing errors and the alignment errors, but not by the design.

\subsection{Further development and optimization}

The current design will be complemented with the optimized ADC, real lens models, the pyramid double prisms and an optional neutral density filter placed nearby the dichroic membrane to protect the camera from too bright NGS.

Note that folding mirror 2 (Fig. 4) could be replaced by an OAP if we want to output the AO corrected beam with another F\#. For instance, we could use a convergent beam for the verification camera.

The design can be slightly modified in order to update it to a GLAO (Ground Layer adaptive Optics) system. Indeed, we can add a beam splitter before the PWFS box and insert several PWFS box around it.

\section{THE PYRAMID PRISM DESIGN}

\subsection{Single prism concept}

Design of the pyramid wave front sensor (PWFS) prisms requires optimization steps on chromatic and pupil distortion errors. Pyramid prism has four facets, so that the prism creates four pupil images on the PWFS detector imaging area. Individual pupil images has a point spread function (PSF) with a certain full width at half maximum (FWHM).

Previously, we considered to use a single prism to create the four pupil images, however we saw that the single prism creates chromatic aberration larger $(>24 \mu \mathrm{m})$ than the PWFS (NUVU EMCCD) pixel size. Additionally, we found that the base angle of a single prism can have small values ( $1^{\circ}$ to $3^{\circ}$ ) which are impractical for the manufacturing.

For these reasons, we opted for designing the PWFS with double pyramid prisms joined at the base. In our design, we aim for easy manufacturing of the prisms and matching glass materials to minimize the chromatic errors. 


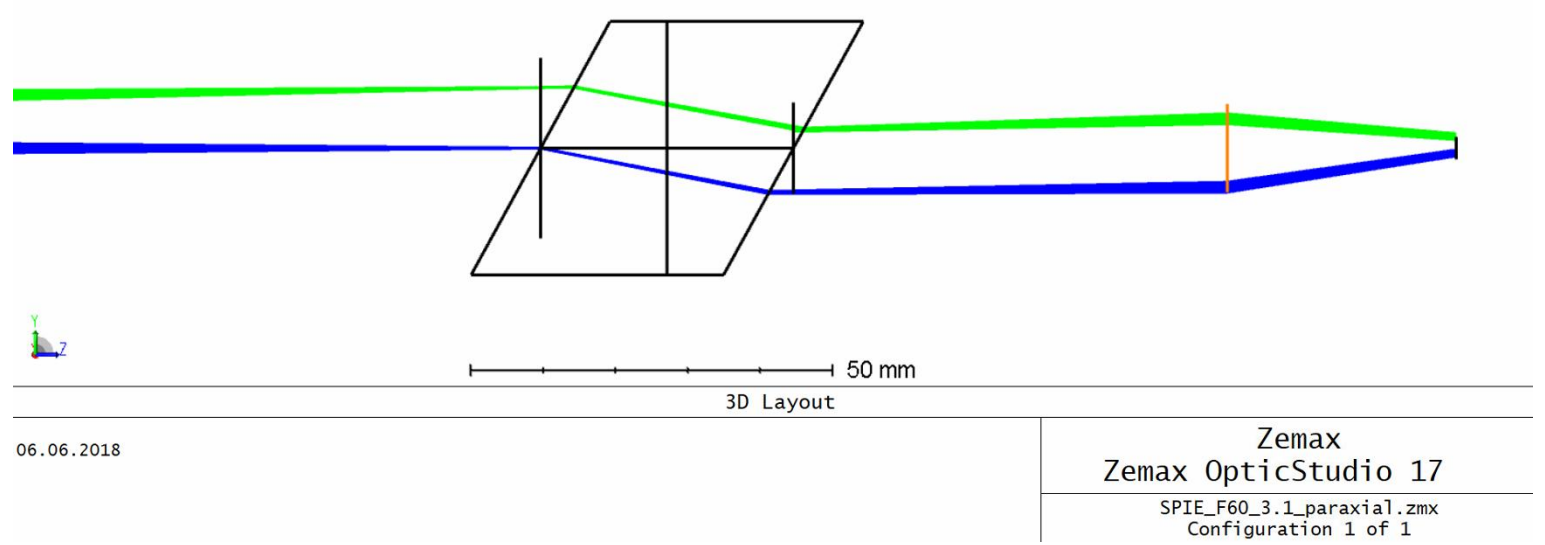

FiguRE 5. 2D layout for the double pyramid prism WFS with paraxial relay lens.

\subsection{Double prism design system parameters}

The overall PWFS design can be seen in Fig. 5. We used ZEMAX OpticsStudio 17 to prepare and optimize the design with a paraxial imaging lens.

All lengths units were specified in millimeters. We used 5 bands of visible light ranging from $0.5 \mu m$ to $0.9 \mu m$ with $0.10 \mu \mathrm{m}$ steps to represent the wavefront sensing range. In order to perform the optimization, we used 2 fields with the on-axis field focused on the pyramid prism while the off-axis field hit the pyramid prism $8 \mathrm{~mm}$ away from the tip. This is to make sure that the pyramid has enough field of view for the natural guide star acquisition (communication from Olivier Lardière). From the previous section, incoming telescope pupil image is focused on the apex of the prism with an F-number of 60.

\subsection{Glass optimization results}

We constrained the semi diameter of individual prisms to be $17.5 \mathrm{~mm}$ to ease the manufacturing process. We solved out the length of the prisms in z-direction to be $17.480 \mathrm{~mm}$ for both pyramids. We used Schott and Ohara catalogues for the glass optimization. We found that the glass pair of L-BAL43/S-BSM14 for the first and second glass gives the minimum lateral color shift seen in Fig. 6. These glasses are produced by Ohara and have low dispersion and medium Abbe numbers. We calculated the base angles of the prisms as the tangents of the surfaces in y-direction. After the optimization, tangent of the first prism is found to be 0.548 (base angle : $28.72^{\circ}$ ) and tangent of the second prism is found to be 0.551 (base angle : $28.85^{\circ}$ ).

\subsection{Prism tip size}

Depending on the telescope $F$-number and wavelength for the wavefront sensing, a nominal value for the tip size of the front pyramid can be calculated. Some percentage of the incoming photon flux is expected to be lost due to the flatness of the prism tip. This causes a drop in the star magnitude to be used for the wavefront sensing. Diffraction limited PSF FWHM should be larger than the width of the pyramid prism tip and the ratio of the PSF FWHM with respect to the tip size gives an approximation of the energy loss.

We calculated the prism tip size for $F / 60$ for the central wavelength of $\lambda=0.6 \mu m$. We found a minimum tip size of $12 \mu \mathrm{m}$ for $\% 50$ energy loss. In the case of excellent atmospheric seeing conditions, we can have a modulation radius of $2 \lambda F$ which should be consistent with the smallest size requirement of the prism tip.

\subsection{Pupil shape and achromatic optimization}

We optimized the resulting pupil images for size, anamorphism and rotation. After the optimization we find that pupil image diameter on the sensor is $0.798 \mu \mathrm{m}$ which corresponds to $\% 19.8$ pupil separation. The footprint diagram given in Fig. 7 shows the image of a single pupil on the wavefront sensor. We analyzed the distortion percentage on the pupil images and found a distortion of $\% 0.0132$ with respect to the pupil diameter. The 


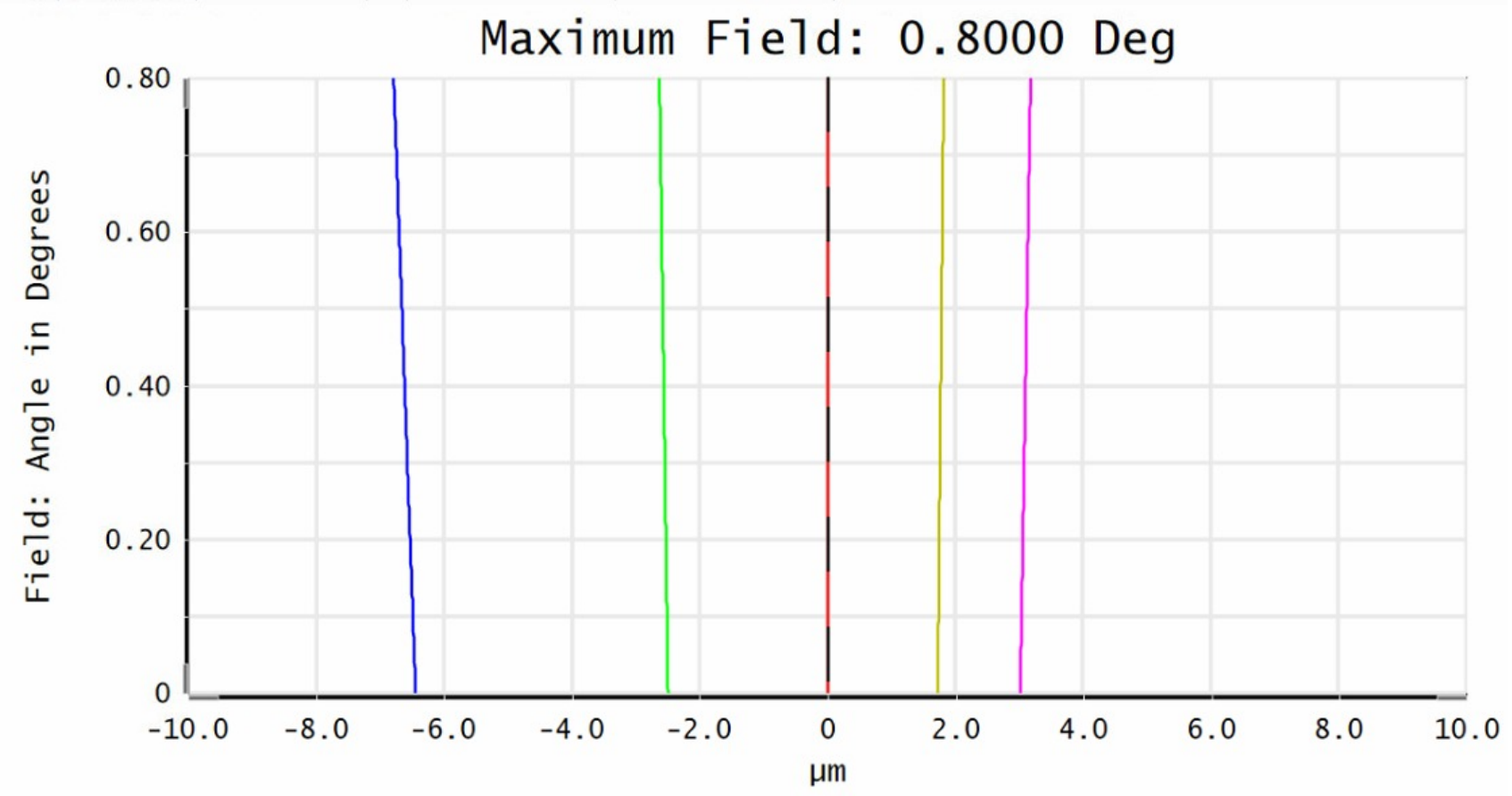

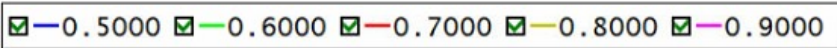

\section{Lateral Color}

06.06 .2018

Data Referenced to Wavelength $0.700000 \mu \mathrm{m}$

Real rays used.

Zemax

Zemax OpticStudio 17

SPIE_F60_3.1_paraxia1.zmx

Configuration 1 of 1

FiguRE 6. Lateral color shift diagram with respect to the wavelength.

TABLE 1. Overall optimization results of the double pyramid prisms.

$$
\begin{array}{l|l}
\text { Prism length } & 17.48 \mathrm{~mm}
\end{array}
$$

Minimum apex size $12 \mu \mathrm{m}$

Base angle of first prism $28.72^{\circ}$

Base angle of second prism $28.85^{\circ}$

Material of first prism L-BAL43

Material of second prism $\quad$ S-BSM14

Pupil image diameter $0.798 \mu \mathrm{m}$

Image separation $19.8 \%$

Lateral color for $0.5 \mu \mathrm{m} \quad 6.46 \mu \mathrm{m}$

Lateral color for $0.7 \mu \mathrm{m} \quad 0.0 \mu \mathrm{m}$

Lateral color for $0.9 \mu \mathrm{m} \quad 3.01 \mu \mathrm{m}$

Pupil distortion $0.0132 \%$ 
chromatic errors on the sensor area is shown in Fig. 6. The lateral color error is relatively constant for the WFS wavelengths with individual shifts of $6.46 \mu \mathrm{m}$ and $3.01 \mu \mathrm{m}$ for the wavelengths of $0.5 \mu \mathrm{m}$ to $0.9 \mu \mathrm{m}$. Lateral color shift error is smaller than the pixel size $(24 \mu \mathrm{m})$ of the WFS camera and also much smaller than the first airy disk $(44 \mu m)$ of the PSFs.

\subsection{Further development}

Overall optimization results of the pyramid prisms and four pupil images on the WFS can be seen in table 1. For future modifications, we plan to design an achromatic relay lens with multiple lenses and optimize the assembly together. Additionally, we plan to tolerance the PWFS design for temperature dependence.

\section{TO CONCLUDE}

We expect the parts of the PWFS to be delivered in October 2018, for mounting and testing in ALPAO premises with the 468 actuators DM. A classical control will be implemented for 1st light, and the loop control will be done with the ACE controller, from ALPAO. Soon after this laboratory demonstration, the optimization algorithm will have to be developed, as well as the AO mechanical bench. First light at the telescope is scheduled for August 2020.

\section{ACKNOWLEDGMENTS}

We would like to warmly thank Paola Spano', Olivier Lardière and Jean-Pierre Véran for the critical help and multiple advices they provided us during this work. We would not have been able to do it without them.

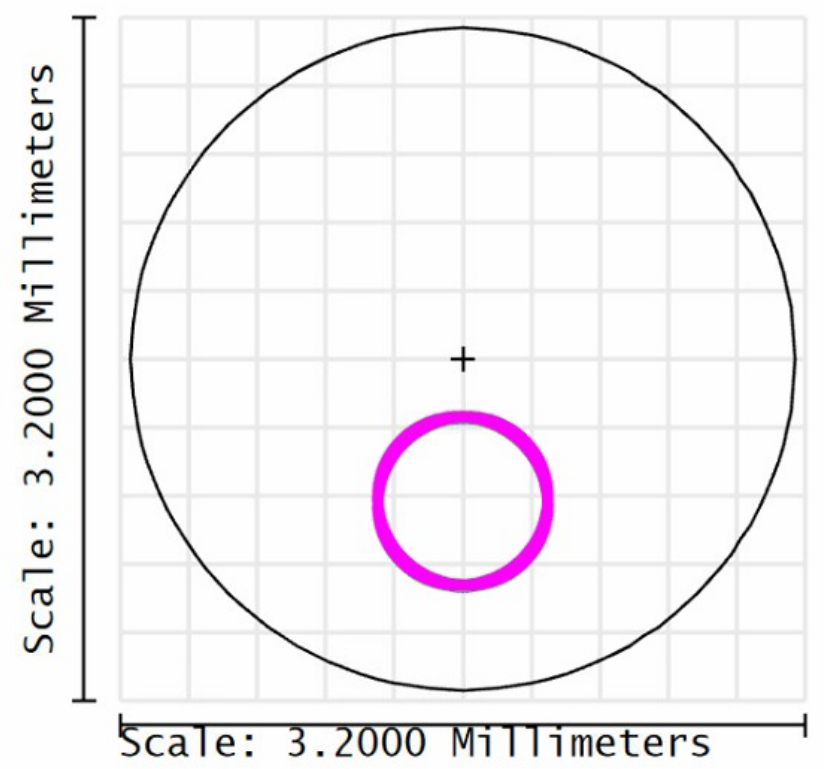

$\square+0.5$

$\square=0.6$

$\square \wedge 0.7$

$\square+0.8$

$\square=0.9$

Aperture Diameter: 3.1000

\begin{tabular}{|c|c|}
\hline Footprint Diagram & \\
\hline $\begin{array}{l}\text { 06.06.2018 } \\
\text { Surface 9: plane of interest } \\
\text { Ray X Min = - } 0.3974 \text { Ray X Max = } \\
\text { Ray } Y \text { Min }=-1.0675 \text { Ray } Y \text { Max }=-0.2674 \\
\end{array}$ & $\begin{array}{c}\text { Zemax } \\
\text { Zemax OpticStudio } 17\end{array}$ \\
\hline $\begin{array}{l}\text { Max Radius }=1.0675 \text { Wavelength }=\text { A11 } \\
\text { Legend items refer to Wavelengths }\end{array}$ & $\begin{array}{l}\text { SPIE_F60_3.1_paraxia1.zmx } \\
\text { Configuration } 1 \text { of } 1\end{array}$ \\
\hline
\end{tabular}

FIGURE 7. Footprint diagram of a single pupil image on the WFS imaging area. 


\section{REFERENCES}

[1] Yeşilyaprak, C., Yerli, S. K., Keskin, O., and Güçsav, B. B., "DAG : a new observatory and a prospective observing site for other potential telescopes," in [Observatory Operations : Strategies, Processes, and Systems VI], Proc. SPIE 9910, 99102U (July 2016).

[2] Ragazzoni, R., "Pupil plane wavefront sensing with an oscillating prism," Journal of Modern Optics 43, 289-293 (Feb. 1996).

[3] Jolissaint, L., Eikenberry, S., Bouxin, A., Keskin, O., and Yesilyaprak, C., "The Flexible Adaptive Optics Concept," in [Adaptive Optics for Extremely Large Telescopes V (AO4ELT5)], (June 2017).

[4] Jolissaint, L., Ragland, S., Christou, J., and Wizinowich, P., "Determination of the optical turbulence parameters from the adaptive optics telemetry : critical analysis and on-sky validation," submitted to Applied Optics (May 2018).

[5] Lardière, O., Pazder, J., Véran, J.-P., and van Kooten, M., "Double-Pyramid Wavefront Sensors : Tolerance Relaxation and Cheaper Alternatives using Achromatic Double-Roof Prisms," in [Adaptive Optics for Extremely Large Telescopes V (AO4ELT5)], (June 2017).

[6] Rigaut, F. and van Dam, M., "Simulating Astronomical Adaptive Optics Systems Using YAO," in [Adaptive Optics for Extremely Large Telescopes III (AO4ELT3)], (May 2013).

[7] Riccardi, A., Bindi, N., Ragazzoni, R., Esposito, S., and Stefanini, P., "Laboratory characterization of a Foucault-like wavefront sensor for adaptive optics," in [Adaptive Optical System Technologies], Proc. SPIE 3353, 941-951 (Sept. 1998). 